\title{
Advantages of Jelly over Liquid Formulations for Pediatrics
}

\section{Doolaanea AA* and Bahari AZBS}

Advanced Drug Delivery Lab, Department of Pharmaceutical Technology, Kulliyyah of Pharmacy, International Islamic University Malaysia, 25200 Kuantan, Malaysia

\begin{abstract}
Jellies for oral administration are non-flowable gelatinous preparations having a certain shape and size. This dosage form is being explored for applications for pediatric patients and other populations like geriatric and dysphagic patients. It has several advantages over liquid dosage forms in term of palatability, patient acceptance, suitability for controlled release applications and stability. Due to lack of information of several aspects of jellies for pediatrics, much more further studies are encouraged.
\end{abstract}

Keywords: Jelly; Pediatric; Formulation; Liquid dosage

\section{Definition of Jellies}

According to the Japanese Pharmacopeia $17^{\text {th }}$ edition (JP XVII) [1], jellies for oral administration are non-flowable gelatinous preparations having a certain shape and size, intended for oral administration. The United States Pharmacopoeia does not have jellies for oral administration. Instead, it prefers to use the term 'gel' which is not to be ingested [2]. This is the definition in USP: 'Jelly (not preferred; see Gel): A semisolid dispersion of small particles or a solution of large organic molecules interpenetrated by a solution containing a gelling agent to promote stiffness.' Jellies are usually prepared by mixing active substance(s) with suitable excipients and polymer gel base, gelatinizing and forming into a certain shape and size by a suitable method [1].

The JP XVII states that jellies for oral administration meet the requirements of dissolution test or show an appropriate disintegration. In addition, jellies for oral administration meet the requirements of 'Uniformity of Dosage Units' [1]. This test is usually required for dosage forms that are packaged in single-dose container. For packaging, JP XVII states that tight containers are usually used for jellies for oral administration; for the preparations that are susceptible to degradation by evaporation of water, a low moisture-permeability container or packaging may be used.

\section{Jellies Compared to Liquid Formulations}

Pediatric oral formulations can be scientifically challenging to develop. The prerequisites for both a measurable dosage form based upon bodyweight and taste-masking are two of the challenges unique for pediatric oral formulations. The majority of active pharmaceutical ingredients (APIs) found in oral dosage forms have bitter taste [3]. Taste masking of liquid formulation presents a major challenge because most of pediatric preparations are syrups and suspensions. Taste masked drug delivery research is gaining importance and commercial success for the quality of treatment provided to suffering patients, especially children [4].

Pediatric population cover the age from birth up to age 16 or 18 . This population can be subdivided according to the document from European Medicines Agency (EMEA) as following [5]:

- Preterm newborn infants.

- Term newborn infants (0-27 days).

- Infants and toddlers (1 month to 23 months).

- Children (2-11 years): could be further subdivided in terms of the child's ability to accept and use different dosage forms, e.g. into preschool children (2-5 years) and school children (6-11 years).
- Adolescents (12-16 or 18 years).

The physicochemical and organoleptic properties of the active drug substance such as solubility, chemical stability, and taste along with the intended dose can determine which formulations are feasible to develop. Strickley [6] reported that oral pediatric formulations are available in 17 different varieties and can be either; ready-to-use formulations such as a (1) solution, (2) syrup, (3) suspension, (4) tablet, (5) scored tablet, (6) chewable tablet, (7) orally disintegrating tablet, or (8) thin strip, or formulations that require manipulation such as a (9) powder for constitution to a suspension, (10) tablet for constitution to a suspension, (11) powder for constitution to a solution, (12) drops for reconstitution to a suspension, (13) concentrated solution for dilution, (14) effervescent tablet, (15) bulk oral granules, (16) bulk oral powder, or (17) solid in a capsule to mix with food or drink.

According to our search, no new pediatric dosage form was commercialized other than those reported in the previous study. A careful view on the 17 dosage forms leads to conclude that the final dosage forms that the pediatric will administer (after reconstitution) can be classified into:

a) Liquid dosage forms: solutions, syrup, suspension, powder for constitution to a suspension, tablet for constitution to a suspension, powder for constitution to a solution, drops for reconstitution to a suspension, concentrated solution for dilution, effervescent tablet.

b) Solid dosage forms: tablet, scored tablet, chewable tablet, orally disintegrating tablet, thin strip, bulk oral granules, bulk oral powder and solid in a capsule to mix with food or drink.

A major concern is at what age children can safely swallow solid oral dosage forms such as tablets or capsules. Again, this is generally a factor of age and health status but there are significant inter-patient differences around an average of about 6 years. Taste, smell and texture will be important factors for any medicine administered orally. Children are unlikely to tolerate repeated administration of medicinal products, which are uncomfortable, painful or stressful [5]. It is estimated that

*Corresonding author: Doolaanea AA, Advanced Drug Delivery Lab, Departmen of Pharmaceutical Technology, Kulliyyah of Pharmacy, International Islamic University, Malaysia, Tel: +60136238628; E-mail: abdalmonemdoolaanea@yahoo.com

Received January 04, 2017; Accepted January 24, 2017; Published February 06, 2017

Citation: Doolaanea AA, Bahari AZBS (2017) Advantages of Jelly over Liquid Formulations for Pediatrics. J Formul Sci Bioavailab 1: 102.

Copyright: ( 2017 Doolaanea AA. This is an open-access article distributed unde the terms of the Creative Commons Attribution License, which permits unrestricted use, distribution, and reproduction in any medium, provided the original author and source are credited. 
$50 \%$ of the population have problem of swallowing tablets, especially the pediatric and geriatric population [7].

Current pediatric dosage forms have several drawbacks especially for the age below 6 years. The ideal pediatric formulation needs to consider the following criteria as suggested by European Medicines Agency [5]:

- Minimal dosage frequency,

- $\quad$ One dosage form fits all or a full range,

- $\quad$ Minimal impact on life style,

- Minimum, non-toxic excipients,

- Convenient, easy, reliable administration,

- $\quad$ Easily produced, elegant, stable,

- Cost and commercial viability.

Current liquid dosage forms provide several advantages such as dose flexibility and ease of swallowing. In fact, they are the most appropriate for younger pediatric patients. However, liquid dosage forms have several disadvantages, among them [5].

- The dose volume is a major consideration:

- Less than $5 \mathrm{ml}$ for children under 5 years

- Less than $10 \mathrm{ml}$ for children of 5 years and older,

- Stability issues: the drug is being in solution or suspension form and hence easier for degradation.

- $\quad$ Patient acceptability: since the drug is completely or partially dissolved it will be in direct contact with the taste sensors in the tongue. This introduces challenges in taste masking and requires high sugar or sweetener concentrations to be used.

- Challenging controlled release: only few sustained release liquid formulations are available in the market.

- Higher transportation costs: due to the large weight of the product.

On the other hand, jellies may address majority of the drawback of the liquid dosage forms. Jellies have higher viscosity than liquid preparations and this property gives jellies advantage in taste masking. Gohel [8] formulated paracetamol in oral jelly (referred as gel in their publication) and found that paracetamol jellies possessed acceptable sensory characteristics when evaluated by human volunteers. Paracetamol is a well-known drug for its very bitter taste. The more palatable the formulation, the higher the dose volume can be tolerated [5]. Therefore, jelly volumes higher than $10 \mathrm{ml}$ might be administered by pediatrics without difficulties.

In addition, controlled release is possible in jelly formulations and it is more practical than liquid formulations. Paracetamol in sustained release jelly formulation was investigated by using carrageenan as gelling agent Miyazaki [9] and other gelling agents like agar, gelatin, gellan, pectin and xyloglucan [10]. In these studies, the sustained release property was achieved through the vehicle itself. By choosing the right gelling agent at suitable concentration, the drug released slowly from the jelly vehicle.
On the other hand, like suspension dosage forms; jellies may be used to suspend drug particle, drug microparticles or pellets. In this case, the sustained release properties may result from the particles themselves and the jelly will serve as a vehicle that carries the particles. In this proposed formulation, the jelly formulation may have advantages over the suspension. The high viscosity of the jellies prohibits the suspended particles from sedimentation.

In term of drug stability, there is lack of information regarding stability of drugs in jelly vehicle or the relation between the drug degradation and viscosity of the vehicle. However, from the concept of chemical reactions, it can be predicted that molecules move slower at higher viscosity leading to slower reaction and so on slower degradation than liquid solutions. More experimental data are needed to verify this anticipation.

\section{Conclusion}

Jelly as an oral dosage form for pediatrics is being explored. It has high potential due to several advantages over liquid formulations in term of patient acceptance, suitability for controlled release applications, stability and other aspects. More studies are needed to further explore this new dosage form.

\section{References}

1. Committee JPE (2016) The Japanese Pharmacopoeia (17thedn) Tokyo, Japan.

2. Convention USP (2016) The United States Pharmacopeia (39thedn) United States Pharmacopeial Convention.

3. Maniruzzaman M (2014) A review on the taste masking of bitter APIs: hot-melt extrusion (HME) evaluation. Drug Development and Industrial Pharmacy 40: 145-156.

4. Kumar KS (2013) Recent trends in taste masking of bitter drugs. J Drug Deliv Res 1: 1-11.

5. EMEA CfMPfHU (2006) Reflection paper: formulations of choice for the paediatric population, L EMEA.

6. Strickley RG (2008) Pediatric drugs-a review of commercially available oral formulations. Journal of Pharmaceutical Sciences 97: 1731-1774.

7. Ayenew $Z$ (2009) Trends in pharmaceutical taste masking technologies: a patent review. Recent patents on drug delivery \& formulation 3: 26-39.

8. Gohel M (2009) Preparation and evaluation of soft gellan gum gel containing paracetamol. Indian Journal of pharmaceutical sciences 71: 120-124.

9. Miyazaki S (2011) Carrageenan Gels for Oral Sustained Delivery of Acetaminophen to Dysphagic Patients. Biological and Pharmaceutical Bulletin 34: 164-166.

10. Miyazaki S (2009) Preparation and evaluation of gel formulations for ora sustained delivery to dysphagic patients. Drug Development and Industrial Pharmacy 35: 780-787. 